Research Article

\title{
The Application of Visual Sensors in the Artistic Design of College Campus Landscape
}

\author{
Xuan Kan ${ }^{1}{ }^{1}$ and Deli Cao ${ }^{2}$ \\ ${ }^{1}$ College of Art, Nantong University, Nantong, Jiangsu, China \\ ${ }^{2}$ LuXun Academy of Fine Arts, Shenyang, China \\ Correspondence should be addressed to Xuan Kan; kan.x@ntu.edu.cn
}

Received 2 September 2021; Revised 6 October 2021; Accepted 8 October 2021; Published 23 November 2021

Academic Editor: Haibin Lv

Copyright (c) 2021 Xuan Kan and Deli Cao. This is an open access article distributed under the Creative Commons Attribution License, which permits unrestricted use, distribution, and reproduction in any medium, provided the original work is properly cited.

\begin{abstract}
The research purpose is to solve the problems of low efficiency, low accuracy, and high cost of traditional environmental landscape mapping and landscape volume measurement methods in the artistic design of college campus landscape and make up the defects that the traditional campus monitoring is vulnerable to adverse weather, which results in low monitoring accuracy. Primarily, a binocular stereo vision measurement based on Scale Invariant Feature Transform (SIFT) matching algorithm is proposed, which can realize accurate collection of environmental spatial information and measurement of landscape volume without contact in the process of campus landscape design. Then, the visual monitoring system of college landscape based on the Internet of Things (IoT) is constructed to realize real-time monitoring and early warning of human damage to campus landscape. The proposed method is verified by actual measurement of different objects and simulation experiments using simulation software. Ultimately, the application of visual sensors in artistic design of college campus landscape is analysed by literature analysis. The results show that (1) the error of the improved binocular stereo vision measurement designed here is $52.32 \%$ and $59.69 \%$ lower than that of the traditional measurement method when measuring the same object with different volumes and the volumes of different objects, respectively, which indicates that the measurement accuracy of the new method is higher. (2) The proposed landscape visual monitoring method based on IoT improves the image recognition accuracy by $21 \%$ compared with the traditional digital image monitoring method. The average recognition time is shortened by $12 \mathrm{~ms}$, which ensures the accuracy and improves the recognition efficiency. (3) Through the analysis of existing literature, it is found that the binocular stereo vision sensor can be used to monitor the whole process of landscape construction in real time. The sensor can be combined with social networks, mobile terminals, and physiological monitoring equipment to comprehensively analyse and evaluate people's preference for campus landscape. The proposed method has broad application prospects in campus landscape design, construction, and maintenance. The research purpose is to provide important technical support for the improvement of the overall image of the college campus and even the city for the design of landscape environment and the technical upgrading of maintenance work in the college campus.
\end{abstract}

\section{Introduction}

The meaning of the existence of colleges and universities is not just to provide a place for students and staff to study and research [1]. Its environment can behave the human spirit, culture ethos, and aesthetic characteristics of campus. Colleges and universities have been gradually opened up to the outside, which makes them important parts of public environment of cities [2]. In these few years, many cities of China have initiated university construction pro- jects on a broad scale. Artistic design of college and university landscape plays an important role in general university construction [3].

But there are still some problems in relative work of artistic design of college and university landscape. A large cost of resource and time exist in prophase work of artificial surveying and mapping. Moreover, on the purpose of ensuring that campus landscape design fits practical need and the purpose of preventing campus landscape from decaying, relative staff make statistical analysis of preference on the 
campus landscape. Usually, they need to collect massive monitoring data and questionnaires or follow the path of themselves. Not only too much manpower and material are cost, but also effect and accuracy cannot get enough guarantee in traditional way, not to mention the impact of bad weather. Vision sensor is cheaper, faster, and easier to use and maintain. It can also be used under bad environment. Most importantly, it can provide objective criteria and intuitive results [4]. At present, the binocular stereo vision sensor (BSVS) is the most widely used sensor in various fields. It is an important technology in 3D shape measurement. Using the known spatial mutual position relationship, two or more cameras obtain the scene image of the same measured object at the same time through the theory of polar geometry and parallax principle to obtain the $3 \mathrm{D}$ geometric information of the measured object, so as to achieve the accurate measurement of the object [5]. Zhang [6] introduced BSVS into the field of health monitoring of Chinese ancient buildings for the overall deformation monitoring process of Chinese ancient buildings. The results of experiments show that this method can automatically and spontaneously obtain the three-dimensional coordinates of the overall deformation points of Chinese ancient buildings without any contact. Li and Zhang [7] proposed a noncontact displacement measurement system based on binocular stereo vision measurement and wireless sensor theory. The experimental results show that the integrated displacement measurement system designed here has high accuracy and good stability. The development of binocular stereo vision measurement technology has brought new opportunities for the development of artistic design of college campus environment.

The traditional binocular stereo vision sensor is fragile to the impact of noise when processing images, resulting in inaccurate measurement results. And it is vulnerable to the influence of weather, light, and other factors and cannot identify the bad behaviors such as damaging the campus landscape. Based on the above problems, a binocular stereo vision measurement method based on the SIFT is proposed, and construction is made on a university landscape vision monitoring system based on Internet of Things (IoT). The application of visual sensors in artistic design of college campus landscape is analysed by literature analysis. The research purpose is to provide important technical support for the design of landscape environment and the technical upgrading of maintenance work of the overall image of the college campus and even of the city.

\section{Materials and Methods}

2.1. Analysis of Problems in Campus Landscape Environment Artistical Design. Landscape design is to accommodate grounds and entities on them in harmony and realize their functions, according to perception on landscape design of Newton, "this can settle people, buildings, urban neighbours, human activities and earth in harmony."

An outstanding and reasonable public artistical design is characterised with humanism, inclusion, respect, culture, communication, etc. [8]. The design should express its psychological and humanism care to the objects in the environ- ment. However, with the proceeding of reform in colleges and universities and expansion of staff and students in campuses, current facilities of the campuses cannot meet up with real requirement. Many colleges and universities expand their campuses into the margin places of cities. But many problems in campus landscape construction occur due to the large scale of campuses and short periods of construction. The problems can be divided into these aspects: (1) lack of psychological balance of public behavior, (2) lack of theme in construction planning, (3) design ignorance of geological characteristics, (4) off-balance of spatial scale, (5) lack of peculiarity against other landscapes, (6) unawareness of landscape biology, and (7) ignorance of cultural nature and educational nature of the campus landscape $[9,10]$. Table 1 shows the detailed contends.

Campuses are not just education places to teach knowledge and skills. They are also living environment where students get their temperament comprehensively cultivated. Campus also functions in passing of human history. Its environment is not same as cultural or commercial environment. It should be elegant, solemn, simple, and natural [11]. More focus on the space interaction between inside and outside should be given into artistic design of campus landscape environment. At the same time, artistic landscape design can make campus environment more special and attractive. The final purpose is that a successful artistic design of campus landscape environment should be making people who work in it feel delighted and joyful. Thus, an active and positive atmosphere can be made to meet potential spiritual requirement of the staff and students [12]. Figures 1 and 2, respectively, show the ideas and principles of the artistic design of campus landscape environment.

2.2. Technique Analysis of Sensors. In the GB/T 7665-2005 General Terminology for Sensors, sensors are defined as an equipment or device that can feel the measured object and put out transformed data according to a certain regulation. Figure 3 shows different functional sensors for five human senses.

A sensor normally consists of sensitive elements, transforming elements, and signal conditioning circuit. Sensitive elements are used to feel the measured object and put out a certain physical quantity related to the object. Sensitive elements are normally made up of some kinds of sensitive materials. Sensitive element is core consistence of a sensor. It is usually named by the input physical quantity, like thermal element, photo element, voltage element, magnetic element, or gas element. Sensitive elements applied in the electronic elements can feel the environment close to or even better than that human can feel. In recent years, with the rapid development of electronic computer and information technology, sensitive elements are applied into wider areas. Sensitive elements cannot get more notice. Transforming elements convert nonelectrical quantities output by sensitive elements into electrical quantity. Signal conditioning circuit converts electric parameters output by transforming elements into digital signals for data collection, process controlling, computing, and other purposes [13]. Figure 4 shows function principles of sensors. 
TABle 1: Problems and their characteristics of campus landscape design.

Problem

Characteristics

(1) Lack of psychological balance of public behavior

(2) Lack of theme in construction planning

(3) Design ignorance of geological characteristics

(4) Off-balance of spatial scale

(5) Lack of peculiarity against other landscapes

(6) Unawareness of landscape biology

(7) Ignorance of cultural nature and educational nature of the campus landscape
Due to the lack of entertainment facilities, cultural expressions, and public and private space to communicate, satisfaction of students and staff on landscape decreases.

There are not geological characteristics or themes of flat construction planning. Forms and modes of landscape panning are single.

Colleges and universities design massive water landscapes in areas short of water, where campuses are ignorant of the local geographical situation and waste resource.

For the vision effect, long-distance axis and large-scale spaces are designed in the landscapes. Feeling of users of campuses is ignored. These kinds of design cause lots of trouble to staff and students even undermine the use of landscape.

Campus cultures are not fully taken into consideration by landscape designers, which makes landscape lack of peculiarities and characteristics of campuses. Campus landscapes tend to be the same.

The original biological balance is broken due to the ignorance of coordination between local environment and plants, even the level of biodiversity declines.

Culture ethos and educative functions are severely undermined because the design is made to chase after fashion and symbolic forms.

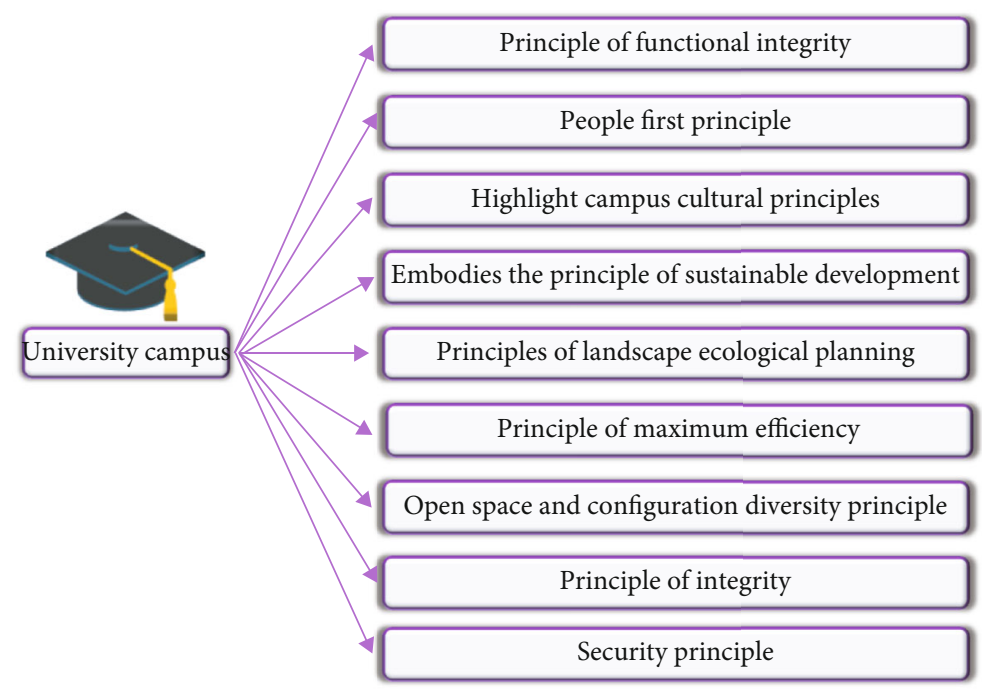

Figure 1: Ideas of artistic design of campus landscape environment.

2.3. Technique Analysis of Vision Sensors. People capture outside information mainly by looking. But the ability of human eyes is limited, and human eyes are vulnerable to outside environment. Firstly, photo elements and imageforming devices capture image data from outside environment. Then, vision sensors convert 2-dimensional images into 1-dimensional electrical signals. The nature of the vision sensor is image processing technique, by capturing signals on the objects' surface and presenting researchers with images.

By difference of the chips, vision sensors can be classified into CCD (Charge Coupled Device) sensors and CMOS (Complementary Metal Oxide Semiconductor) sensors in general. CCD uses the quantity of electric charge to represent the quantity of signals and transport signals in the coupling method. Its functions include photoelectric conversion and information storage and transportation. There are many advantages in CCD, such as auto scanning, feeling of wide spectrum, lightly distorting, small volume, light weight, low system noises, less power loss, high durability, and high reliability. It can be well integrated with other elements. Linear array CCD can capture 1-dimensional images, and planar array CCD can capture 2-dimensional images, and thus, CCD sensors are always applied in industries producing [14]. According to using light source or not, vison senses can be divided into passive light vision and active light vision. CMOS is developed mainly because of its small volume, small power consumption, and low cost. But its imaging quality is a bit lower than CCD [15]. Usual digital products in the market are adopted of CCD or CMOS primarily. Low-class cameras are mainly adopted of CMOS, and the high-end cameras are adopted of CCD. Figures 5 and 6 show the principle and structure of a vision sensor. 


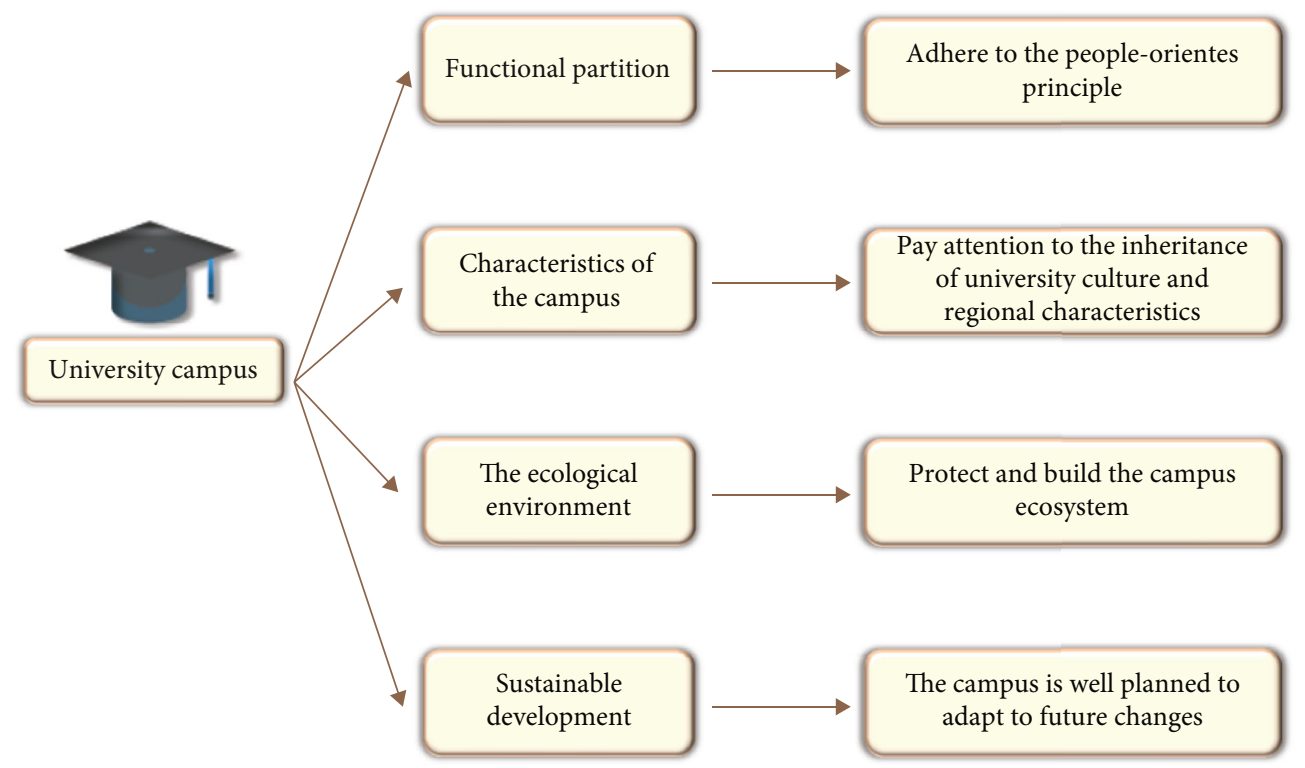

FIGURE 2: Principles of artistic design of campus landscape environment.

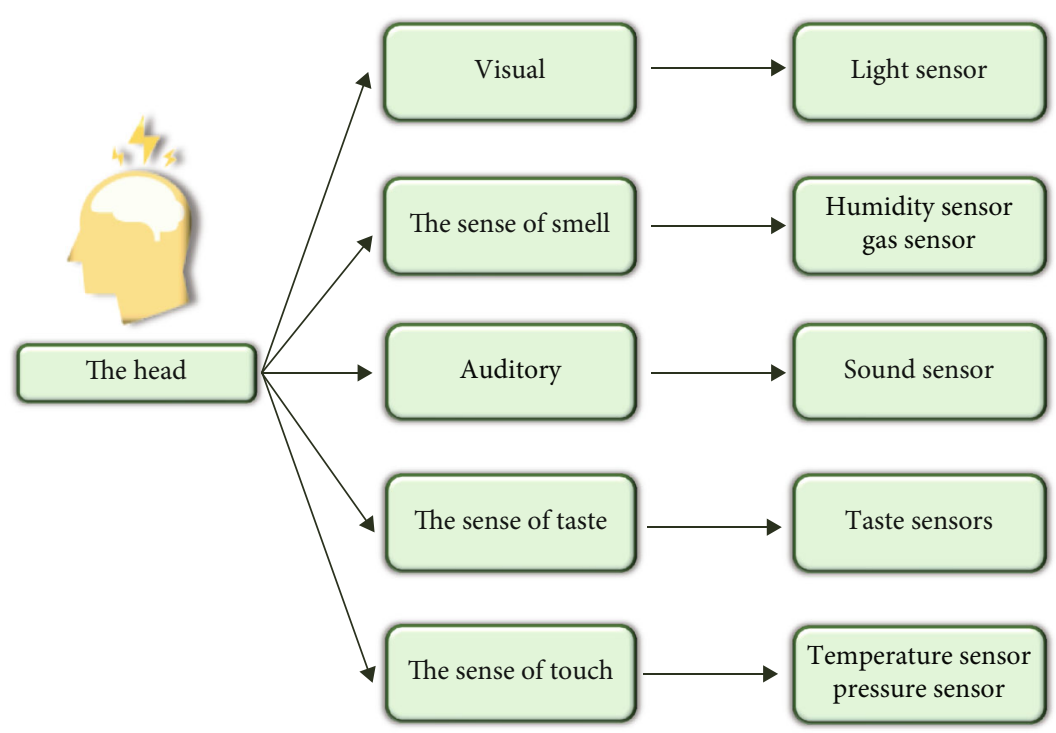

FIGURE 3: Different functional sensors for five human senses.

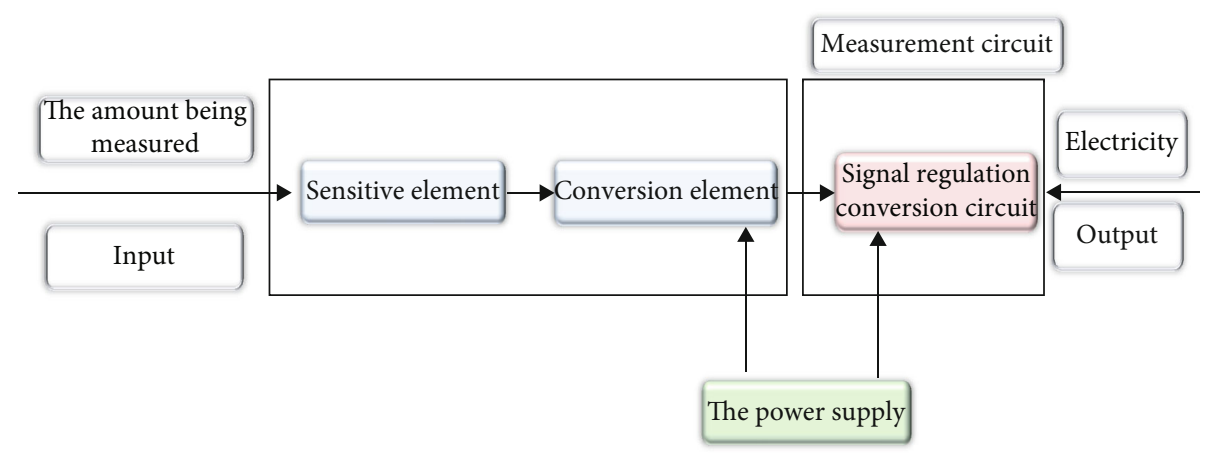

FIGURE 4: Function principles of sensors. 


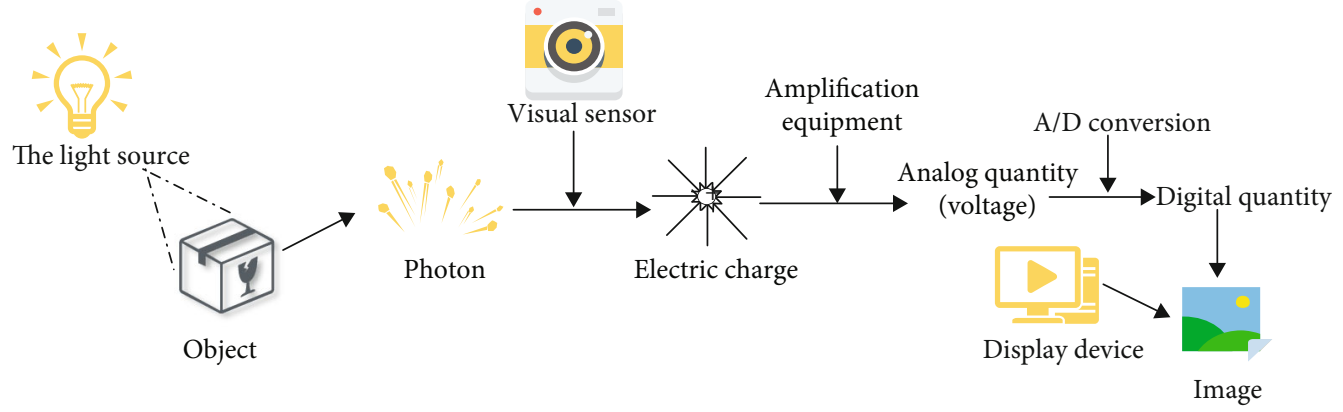

Figure 5: Principle of a vision sensor.

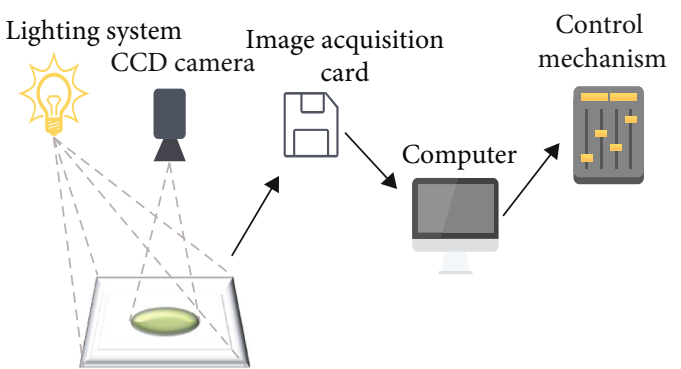

Detected target

Figure 6: Structure of a vision sensor.

\subsection{BSVS Measurement Based on SIFT Matching Algorithm}

\section{(1) Transforming of coordinate axis}

Based on parallax principle, BSVS is an important technology in 3D (3 dimensional) shape measurement [16]. It uses mutual relations of space locations or several cameras to capture the scene figure of the measured principles of epi-polar geometry and parallax. Then, through the scene figures, 3D geometry information of the measured can be calculated. Figure 7 shows the principle of 3D measurement with BSVS.

In Figure 7, $P(x, y, z)$ represents a certain point in the space. Left and right views represent two front imaging planes of the camera, respectively. $O_{1}\left(a_{1}, 0,0\right)$ and $O_{2}\left(a_{2}\right.$, $0,0)$ are the optical centers of the left and right cameras. $P_{1}\left(x_{1}, y_{2}, z_{3}\right)$ and $P_{2}\left(x_{2}, y_{2}, z_{2}\right)$ are the two points mapped from $P$ to the left and right imaging planes. And $f$ represents focal length. $B$ represents the baseline distance, which is the center distance of the left and right camera. $A$ represents the distance from point $P$ to the camera imaging plane.

Through Similar Triangle Principle, Equation (1) is obtained.

$$
\frac{f}{z}=\frac{x_{1}}{x}=\frac{y_{1}}{y}=\frac{x_{2}-x_{1}}{a_{1}}
$$

Then,

$$
x=\frac{a_{1} x_{1}}{x_{2}-x_{1}}
$$

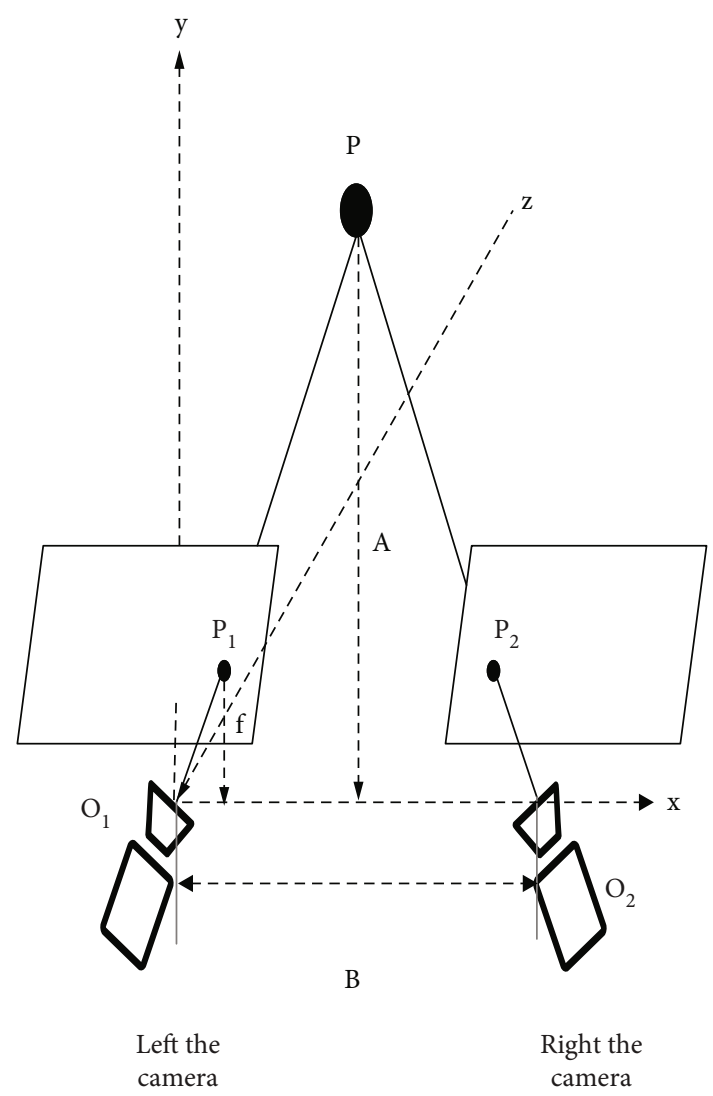

FIgURE 7: Principle of distance measurement with BSVS.

$$
\begin{aligned}
& y=\frac{a_{1} y_{1}}{x_{2}-x_{1}}, \\
& z=\frac{a_{1} f}{x_{2}-x_{1}} .
\end{aligned}
$$

Disparity is represented by $x_{2}-x_{1}$, in Equations (1)-(4).

The binocular camera needs to be calibrated before use. The purpose is to determine the one-to-one correspondence between the position of a certain point of the object in the world space and the pixels in the image and to establish the geometric model of the imaging. The work is to transform the world coordinate system into the camera coordinate system, then into the image coordinate system, and 
finally into the pixel coordinate system. The relationship between the point $(X, Y, Z)^{T}$ in the world coordinate system and the corresponding point $(u, v)$ in the pixel coordinate system is as follows:

$$
s\left[\begin{array}{c}
u \\
v \\
1
\end{array}\right]=\left[\begin{array}{cccc}
a & 0 & u_{0} 0 \\
0 & b & v_{0} & 0 \\
0 & 0 & 1 & 0
\end{array}\right]\left[\begin{array}{cc}
R & T \\
0^{T} & 1
\end{array}\right]\left[\begin{array}{c}
X \\
Y \\
Z \\
1
\end{array}\right]=M_{1} M_{2} \vec{X},
$$

where $R$ is the rotation matrix, $T$ is the transposed matrix, $s$ is the scale factor, $M_{1}$ is the internal reference matrix of camera, $M_{2}$ is the external reference matrix, $a=f / d x, b=f$ $/ d y$, and $d_{x}$ and $d_{y}$ are physical dimensions of each pixel in $x$ and $y$ axes.

\section{(2) Scale Invariant Feature Transform}

Scale Invariant Feature Transform (SIFT) is an image local feature extraction algorithm based on multiscale space. The image feature extracted by the SIFT algorithm is a local feature of scale invariant coordinates, and the affine deformation in the imaging process is eliminated by using the normalized correlation method. It is invariant to rotation, scale scaling, and brightness change and also stable to a certain extent to angle change, affine transformation, and noise. It has good uniqueness and abundant information and is suitable for fast and accurate matching. It also has multivolume lines, high speed, and scalability.

\section{(1) Spatial extreme point detection}

The selection of extreme points is the premise of feature point matching. The image is convolved by Gaussian filters at different scales, and then, the key points are found by using Gaussian filters to blur image differences continuously. The key points are the maximum and minimum Difference of Gaussians (DoG) at different scales. DoG at different scales $D(x, y, \sigma)$ can be expressed as follows:

$$
D(x, y, \sigma)=L(x, y, k \sigma)-L(x, y, \sigma) .
$$

In Equation (6), $\sigma$ refers to the scale parameter. Under the condition of scale $k \sigma, L(x, y, k \sigma)$ is obtained by convolution of the original image $I(x, y)$ with Gaussian blur $G$ $(x, y, k \sigma)$, where $G(x, y, k \sigma)$ is a scale-variable Gaussian function:

$$
G(x, y, \sigma)=\frac{1}{2 \pi \sigma^{2}} e^{-\left(x^{2}+y^{2}\right) / 2 \sigma^{2}} .
$$

\section{(2) Accurate location of key points}

The gradient direction distribution characteristics of adjacent pixels of key points are used to specify direction parameters for each key point, so that the operator has rota- tion invariance. The gradient $m(x, y)$ and direction $\theta(x, y)$ of the image $L(x, y, \sigma)$ after Gaussian blur processing at the size of $\sigma$ can be calculated by the adjacent pixel values:

$$
\begin{aligned}
& m(x, y)=\sqrt{(L(x+1, y)-L(x-1, y))^{2}+(L(x, y+1)-L(x, y-1))^{2}} \\
& \theta(x, y)=a \tan 2\left(\frac{(L(x, y+1))}{(L(x+1, y)-L(x-1, y))}\right) .
\end{aligned}
$$

In the equation, the scale used by $L$ is the scale of each key point. $L(x-1, y), L(x+1, y), L(x, y-1)$, and $L(x, y+1)$ are adjacent pixel values. Parameter $a$ is constant.

\subsection{Analyses on Methods of Landscape Visual Monitoring in Campus}

(1) Principle of college landscape visual monitoring based on IoT

In order to prevent the landscape in colleges from being intentionally damaged, college usually installs monitoring equipment everywhere in the campus for observation. But in severe weather conditions, monitoring will be seriously disturbed, thereby reducing the quality of monitoring images and monitoring signals. Therefore, construction is made on a university landscape visual monitoring system based on IoT. IoT is a network composed of things, where things connect with each other through the network. Based on the Internet, it realizes remote monitoring and management through the combination of GPS technology, sensor technology, and infrared technology within a certain communication specification. A variety of visual sensing monitoring equipment can be set up in colleges to perceive the behavior and action characteristics of the crowd around the landscape. The detected information is transmitted to the monitoring and management personnel through IoT, which judges and processes the received information and provides protection for landscape. The visual monitoring system of college landscape based on the Internet of Things can realize the integration of IoT and monitoring data, replace manual rapid judgment by behavior automatic identification system, and control relevant subsystems to make alarm when bad behaviors are detected. The system mainly includes the following parts. The first is the visual sensors installed everywhere in the school. The second is the visual image analysis server, connected with the visual sensor. The third is the behavior automatic tracking sensor. Among them, the visual image analysis server is the core part of the system, which can accurately identify and match the behavior of people in the monitoring image through relevant algorithms, so as to achieve the accurate monitoring of campus landscape.

(2) Realization of the visual monitoring system of college landscape

The visual sensors in IoT are used to collect the behavior images of the visitors, read the contour images of people, and perform Fourier transform on the center of the behavior 
contour images. The transform results are transformed into symbolic sequences. The hidden Markov model classifier is used to identify and classify the behavior of the visitors, and finally, the accurate monitoring of the campus landscape is realized. The specific methods are as follows: The Fourier Descriptor can be used to project the pixels $\left(x_{i}, y_{i}\right)$ of the contour curve of people's behavior in the image into a complex plane, and an ordered sequence is obtained: $z_{i}=x_{i}+j y$. Equation (9) can be used to discrete Fourier transform of $z_{i}$.

$$
a_{n}=\frac{1}{N} \sum_{i=1}^{N} z_{i} \operatorname{epx}\left(\frac{-j 2 \pi n i}{N}\right) \text {. }
$$

Due to the huge complex operation in the above transformation, it is necessary to use resampling method to process the contour image of tourists' behavior. Resampling is used mainly to the contour image according to the method of equal spacing length as shown in Equation (10).

$$
a_{n}=\frac{1}{N} \sum_{i=1}^{N} r_{i} e p x\left(\frac{-j 2 \pi n i}{N}\right) \text {. }
$$

$N$ is the number of pixels in behavior contour image; $i=1,2, \cdots, N ; r_{i}$ is the radius from any point in the behavior contour curve to the centroid; Fourier coefficients are normalized to get Fourier descriptors:

$$
f=|| \frac{a_{2}}{a_{1}}|,| \frac{a_{3}}{a_{1}}|,| \frac{a_{4}}{a_{1}}|, \cdots \bullet,| \frac{a_{N}}{a_{1}}|| .
$$

The recognition and classification model of behavior can be described by $\lambda=(\pi, A, B)$, where $\pi$ is the initial probability distribution matrix of behavior state, $A$ is the state transition matrix of tourist behavior, and $B$ is the state output probability matrix of behavior.

The initial state probability matrix of behavior in hidden Markov model is set to $\pi=(1,0, \cdots, 0)$, and the initial value of matrix $A$ is as follows:

$$
A=\left[\begin{array}{ccccc}
a_{11} & a_{12} & 0 & \cdots & 0 \\
0 & a_{22} & a_{23} & \cdots & 0 \\
0 & 0 & a_{33} & \cdots & 0 \\
\vdots & \vdots & \vdots & \cdots & \vdots \\
0 & 0 & 0 & \cdots & a_{N N}
\end{array}\right]
$$

The sum of each row in matrix $A$ equals 1 , and $a_{11}=$ $a_{12}, a_{22}=a_{23}, \cdots, \mathrm{a}_{N-1 N-1}=\mathrm{a}_{N-1 N}$.

\subsection{Methods of Experiment}

(1) Methods of experiment of vision measurement verification by binocular stereo

Xiaomi binocular camera and OpenCV 4.1.2 on the Visual Studio 2017 are adopted to collect left and right views, in this experiment. Then, subsequent image informa-

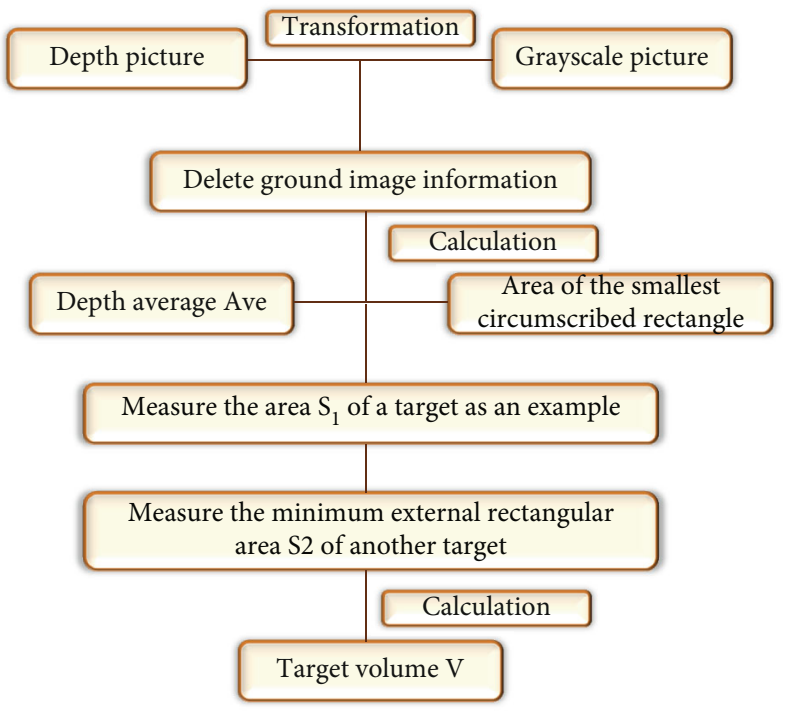

Figure 8: Steps of processing depth picture.

tion and object volume need to be processed and calculated. The camera is placed at a fixed height $H$ to take image of the object placed on the ground. The depth map can be obtained by calculating the parallax information of the image and combining the internal and external parameters. The volume of the object can be obtained by processing the depth map. Two groups of experiments are carried out. Four boxes of different volumes are selected for group 1. Regular objects with different volumes and structures to be tested for experiments are chosen for group 2, including boxes, cylinders, acoustic enclosure, round tables, and books. The improved BSVS measurement designed here is used to measure the volume of the above objects, and the measurement results are compared with the traditional binocular measurement method to calculate the measurement error and explore its effectiveness. Figure 8 shows the steps of processing depth picture.

(2) Test on the visual monitoring system of college campus landscape based on IoT

In order to verify the effectiveness of the monitoring system designed here, the simulation software Matlab 7.1 is used to construct the experimental environment. Concurrently, the traditional digital image monitoring method is selected, and the accuracy and speed of image recognition and classification of visual monitoring are taken as the indexes to conduct a comparative experiment. Two methods are implemented to collect 100 monitoring images, and each image size is $640 * 480$ pixels. The experimental platform is a computer with configuration of Inter core (TM) 2 Duo T9800, 2.8 GHz, DDR3 $10664 \mathrm{G}$, and Windows 7. The experiment is repeated for 100 times to analyse the data generated from the experimental results.

\section{Result}

3.1. Experiment Results of Binocular Stereo Visual Measurement. The improved binocular stereo vision 

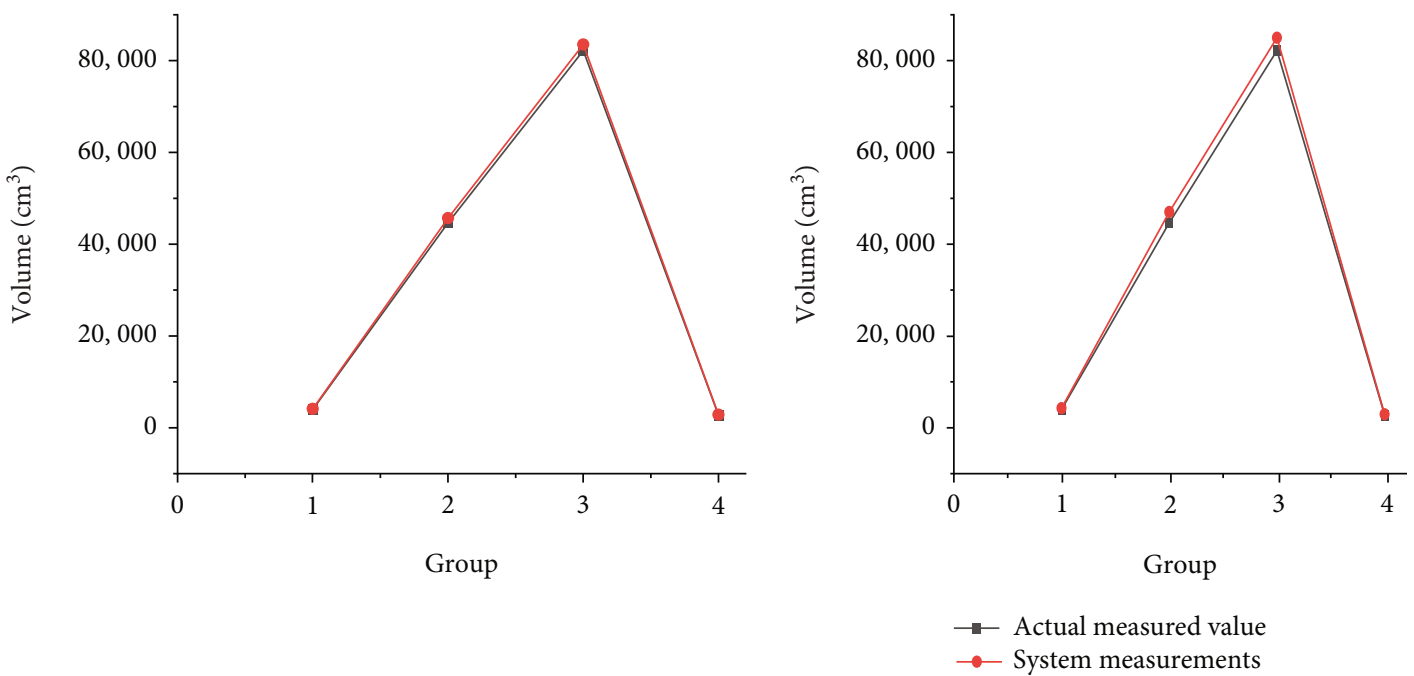

(a)

(b)

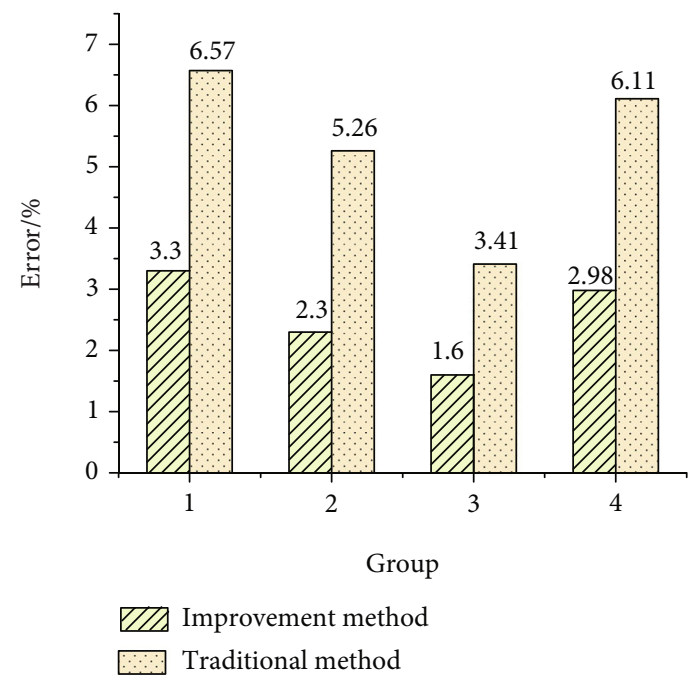

(c)

FIGURE 9: Measurement results and error comparison. (a) Results of the binocular stereo visual measurement; (b) results of the measurement with traditional method; (c) measurement error comparison.

measurement designed here and the traditional method are used to measure the objects of group 1. Figure 9 signifies the comparison of error.

By observing the Figure 9, the error rates of improved binocular stereo vision measurement are 3.3\%, 2.3\%, $1.6 \%$, and $2.98 \%$, respectively, and the average error rate is $2.54 \%$. The error rates of traditional measurement method are $6.57 \%, 5.26 \%, 3.41 \%$, and $6.11 \%$ separately, and the average error rate is $5.34 \%$. The error of the improved binocular stereo vision measurement is about $52.32 \%$ lower than that of the traditional method. Figure 10 displays the measurement results and error comparison of group 2 objects by two methods.

Figure 10 indicates that the volume error rates of different objects measured by improved binocular stereo vision measurement method are $3.25 \%, 3 \%, 3 \%, 5 \%$, and $1.82 \%$, respectively, and the average error is $2.01 \%$. The error rates of traditional measurement methods are 5.35\%, 9.08\%,
$4.43 \%, 9.40 \%$, and $3.28 \%$, respectively, and the average error rate is $5 \%$. The error of the improved binocular stereo vision measurement method is about $59.69 \%$ lower than that of the traditional method.

3.2. Comparison between Traditional Algorithm and Improved Algorithm for Behavior Monitoring Image Recognition. The traditional algorithm and the improved algorithm are used to recognize the behavior monitoring image, respectively. After the 100 experiments are analysed, results are as shown in Figure 11.

Figure 11 shows that in 100 experiments, the recognition accuracy of the traditional algorithm and the improved algorithm for the behavior monitoring image is $76 \%$ and $97 \%$, respectively. The image recognition rate of the improved algorithm is increased by $21 \%$, which is significantly higher than that of the traditional algorithm. The average recognition time of the two algorithms is $25 \mathrm{~ms}$ and $13 \mathrm{~ms}$, 


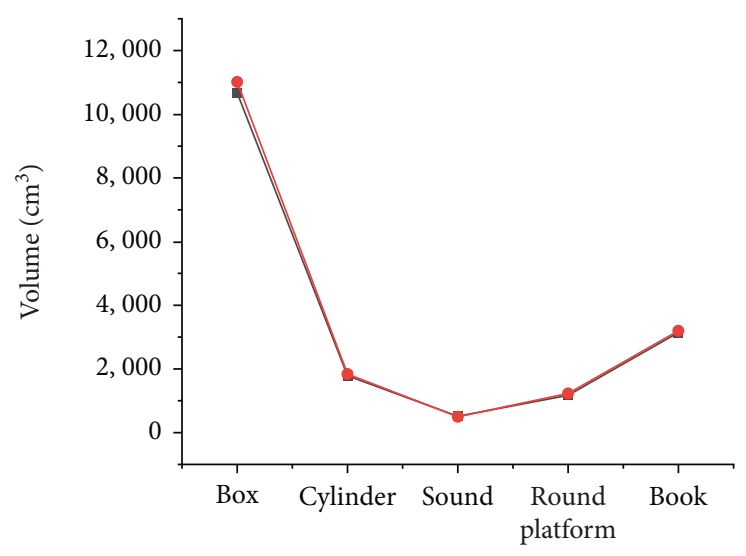

Different objects

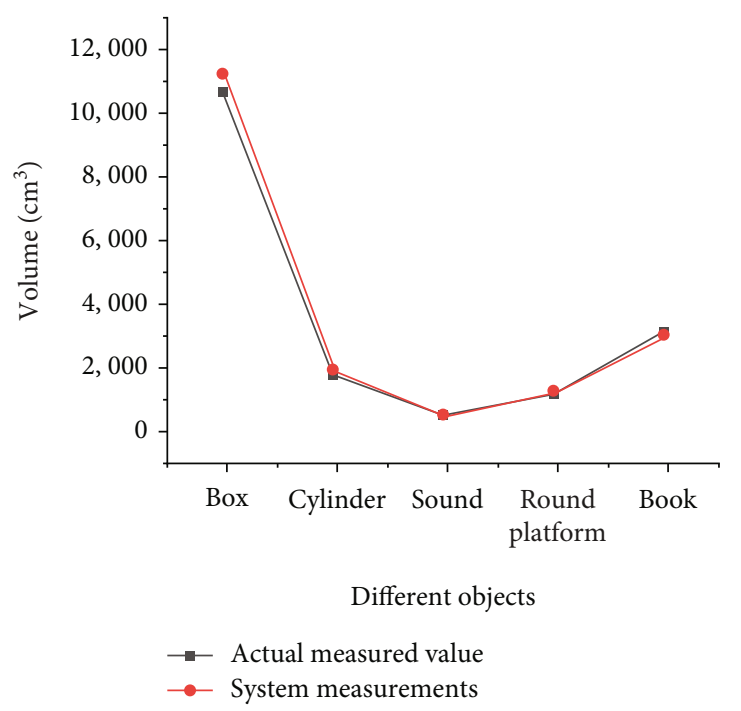

(b)

(a)

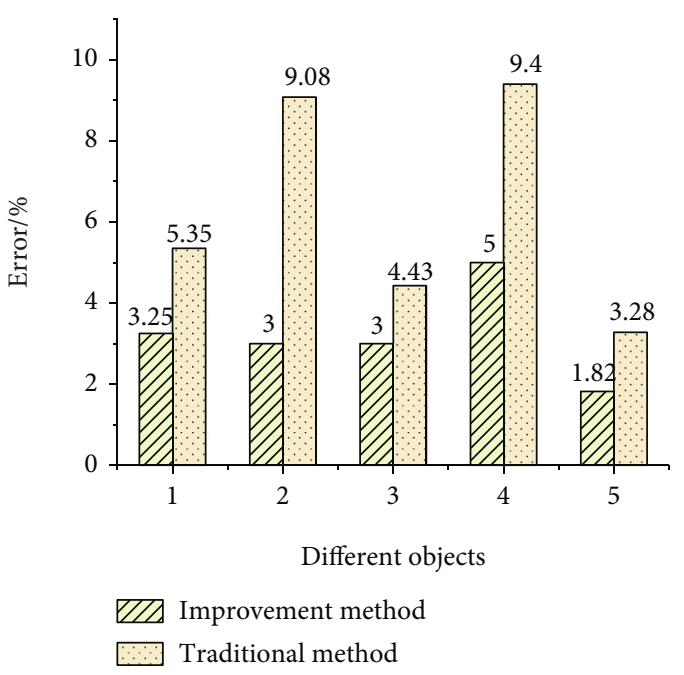

(c)

Figure 10: Measurement results and error comparison of group 2. (a) Results of the binocular stereo visual measurement; (b) results of the measurement with traditional method; (c) measurement error comparison.

respectively. The average recognition time of the improved algorithm is shortened by $12 \mathrm{~ms}$.

In summary, with the binocular vision measurement method based on the SIFT matching algorithm designed here, when measuring the same object with different volumes and the volume of different objects, the error is $52.32 \%$ and $59.69 \%$ lower than the traditional measurement method, namely, the measurement accuracy of this method is higher. It has broad application prospects in the collection of environmental information and landscape volume measurement in artistic design of college campus landscape. The image recognition accuracy of the proposed landscape visual monitoring method based on IoT is $21 \%$ higher than that of the traditional digital image monitoring method. The average recognition time is shortened by $12 \mathrm{~ms}$, which ensures the accuracy and improves the recognition efficiency. It provides support for real-time monitoring and early warning of campus landscape and ensures landscape safety.

3.3. Application Analyses of Vision Sensors in Artistic Design of Campus Landscape and Building. Before conducting artistic design work of campus landscape environment, designer should generally grasp the basic situation of campus environment including planar graphs and detailed information of their sizes. Firstly, designer carries out a thorough exploration and research analyses to plan a reasonable land scape design [17]. Then, blueprint and construction drawing can be made according to the design. Finally, builders begin working on the construction. Figure 12 shows a planar graph of a campus.

Dong et al. held that traditional landscape artistic designs depended on the designers' experience somehow. Besides, there are some uncertainty in actual effect of 


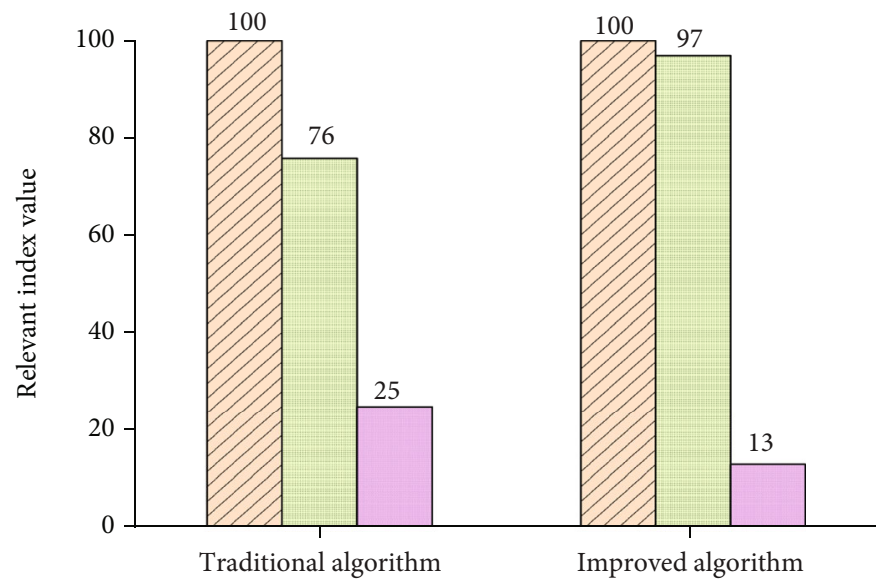

Different algorithms

Number of experiments

Recognition Precision/\%

Average recognition time/ms

FIGURE 11: Experiment data comparison between traditional algorithm and improved algorithm.

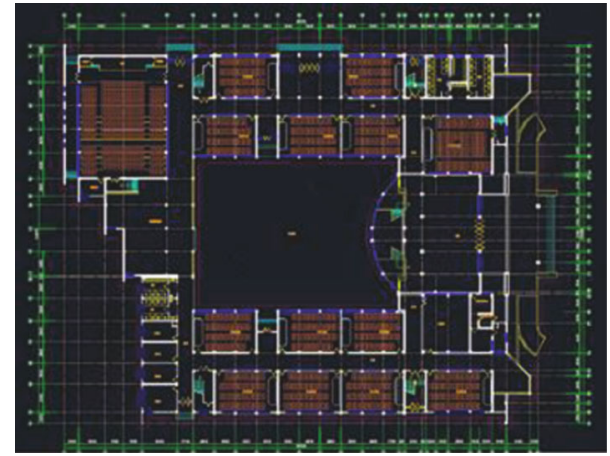

FIGURE 12: Planar graph of a teaching building in a campus.

landscape due to the discrepancy of technique between designers and builders of the landscape [18]. If building of landscape is not reasonable, there will be some potential security risks in campus. Thus, after the landscape building accomplishes, it is necessary to strictly measure and examine the construction. But strong artistic landscapes are usually seen in campuses. These landscapes cover large ground, and their shapes are irregular. Artificial measurement on this kind of landscapes is hard to make, and the accuracy is hard to guarantee. However, vision sensors can process graphs captured by cameras. They can calculate main quantities of the object such as area, center of gravity, length, and location. Data and analysis results are given by them in electricity signal [19]. They can be described as handy, accurate, cheap, and fast.

A $3 \mathrm{D}$ reconstruction method is proposed, to simplify the process of $3 \mathrm{D}$ reconstruction in vision sensors of rotary scanning line structure. Compared with the traditional method of $3 \mathrm{D}$ reconstruction in vision sensors of structured line, substitution of cloud pixels into synthetic depth image is adopted by this new method, which is handy and flexible. With this method, effect of reconstruction of structured line vision sensors is improved to meet the need of $3 \mathrm{D}$ reconstruction in common scenes [20].

A measurement method of the object volume is proposed, based on binocular stereo vision and image processing. First, the BSVS camera shall be marked. Then, two pictures of the object which needs to be measured are taken by two sides of the camera. Thirdly, disparity and depth information can be obtained through processing of the grey scale image from depth information of the picture. Finally, the volume of the object can be calculated with the extraction of the rectangle area and relative height of the object. Results of the experiment suggest that this method of volume measurement can reach the accuracy standard in a certain degree [21].

Furthermore, applying the BSVS technology to the artistic design of college and university landscape according to the practical necessities can make the overall work more effective and accurate. At first, BSVS can capture the 3D images and measure the accurate information of 3D spaces in campus, in the earlier measurement and drawing work. Designers can make design and plan according to the accurate data and spatial images analysed by computer. Secondly, relative data of landscape blueprint and construction drawing can be loaded in the sensors as reference images preliminarily in the campus landscape construction. With comparison and analyses of real scene images and reference images by BSVS, designer can monitor the construction progress live. Thus, problems of landscape construction can be solved in time, such as disparity between actual scenes with anticipated scenes or big difference in the appearance. Light, weather, distance, and temperature cause less influence on vision sensors than on human eyes, when capturing pictures. Besides, BSVS can make accurate measurement on the big irregular landscape. It has a broad applying foreground in the artistic design of college and university landscape environment. 
3.4. Application Analyses of Vision Sensors in Preference Study of the College and University Landscape. "Beauty lies in the eyes of the viewer" says a western scholar. Comprehensions of the beauty lying in things are different to different people. Different visual effect appears in different landscape, and landscape preferences of people are also different. As important methods of judging landscape, landscape preferences are usually used to evaluate the landscape type preference of people. Visual effect, personal need, circumstance, and characteristics of landscape can cause influence on viewer's preference [22]. Preference study of college and university landscape plays an important role in promotion of satisfaction of stuff and students as well as environment of campus. Currently, research of landscape preference is carried out by combination of survey quest and eye movement technology.

Making the landscape of high-tech industry park as the research object, preference evaluation quests from 100 tourists of 40 pictures of landscape are collected and analysed. Thus, the exploration of preference on this kind of landscape is made, according to which, making survey quests to study preference on the landscape pays lots of power, time, and materials [23]. Based on lots of document, it is proposed that combination of eye movement technology with survey quests or depth interview is the necessary basis of perception analyses of human. But eye moment technology on real scene is fragile to environmental influence, and it is hard to design experiment or to obtain samples [24]. A survey on the college students touring in the Tang-Jia Ancient Town is made in eye movement experiment, from the consumer behavior analysing model. In conclusion, landscape types of the tourism destination, abundancy of colours, and the ageing degree can cast influence of the perception of tourists [25].

In recent years, big data information of landscape architecture is obtained through social network, mobile terminal, and physical monitoring. The former two methods can be used to get time and location information, and the latter can be used to capture physical reaction of people viewing different landscape. They are combined to analyse landscape preference [26]. In the preference research of campus landscape, if only the vision sensor or only the digital technology is used, it will be unobjective or unstable. Thus, maybe vision sensors and digital technology can be combined into preference research of campus landscape. Pausing time of tourists before different landscape and people's moving track can be collected through social network and mobile terminal. Getting rid of the influence from unknown factors, collective behavior image can be captured through each vision sensor in campus. Finally, a conclusion can be drawn through the comprehensive analyses and evaluation of the preference on the campus landscape. This conclusion can be referred to promote the actual need of the staff and students as well as comprehensive environment quality in campus.

\section{Conclusions}

The artistic design of college campus landscape includes environmental landscape mapping, scheme design, construction effect monitoring, and landscape maintenance. Usually, jobs in these sections are carried out by manual measurement, maintenance, and installation monitoring, which have the disadvantages of low efficiency, low accuracy, and many other influencing factors. Based on the above problems, a binocular stereo vision measurement method based on the SIFT matching algorithm is proposed, and construction is made on a college landscape vision monitoring system based on IoT. The proposed method is verified by actual measurement experiments and simulation software. The application of visual sensors in artistic design of college campus landscape is analysed by literature analysis. The results show that the error of the binocular stereo vision measurement method designed here is $52.32 \%$ and $59.69 \%$ lower than that of the traditional measurement method when measuring the same object with different volumes and the volumes of different objects, separately, indicating that the measurement accuracy of the method is higher. The image recognition accuracy of the proposed landscape visual monitoring method based on IoT is $21 \%$ higher than that of the traditional digital image monitoring method. The average recognition time is shortened by $12 \mathrm{~ms}$, which ensures the accuracy and improves the recognition efficiency. Through the analysis of existing literature, it is found that the BSVS can be used to monitor the whole process of landscape construction in real time. It is combined with social networks, mobile terminals, and physiological monitoring equipment to comprehensively analyse and evaluate people's preference for campus landscape. The method proposed here has broad application prospects in campus landscape design, construction, and maintenance. The deficiency lies in that the selected measurement objects are generally small in volume, and their adaptability in large-scale landscape volume measurement needs to be further studied. The research purpose is to provide important technical support for the improvement of the overall image of the college campus and even the city for the design of landscape environment and the technical upgrading of maintenance work in the college campus.

\section{Data Availability}

The data used to support the findings of this study are available from the corresponding author upon request.

\section{Conflicts of Interest}

The authors declare no conflicts of existing interest.

\section{References}

[1] L. Lin and Y. Dong, "A study on the campus landscape design of the national university of Singapore," Conference Series: Materials Science and Engineering, vol. 301, no. 1, article 012152, 2018.

[2] Z. Wei, S. Sun, and X. Ji, "The inspiration of rainwater utilization of foreign sponge campus landscape planning for Beijing," IOP Conference Series: Earth and Environmental Science, vol. 227, no. 5, article 052019, 2019.

[3] J. Wu, "Preliminary study on the landscape design of sponge campus based on LID concept-a case study of landscape design in Wuhan University of Technology," IOP Conference 
Series: Earth and Environmental Science, vol. 621, no. 1, article 012109, 2021.

[4] A. Mosaif and S. Rakrak, "A new system for real-time video surveillance in smart cities based on wireless visual sensor networks and fog computing," Journal of Communications, vol. 16, no. 5, pp. 175-184, 2021.

[5] H. Yin, Z. Ma, M. Zhong et al., "SLAM-based self-calibration of a binocular stereo vision rig in real-time," Sensors, vol. 20, no. 3, p. 621, 2020.

[6] C. Zhang, "Overall deformation monitoring of Chinese ancient buildings based on binocular stereo vision," Proceedings of 2021 Academic Exchange Conference on Industrial Architecture (Volume I), vol. 3, no. 1, p. 6, 2021.

[7] H. Li and B. Zhang, "Application of integrated binocular stereo vision measurement and wireless sensor system in athlete displacement test," Alexandria Engineering Journal, vol. 60, no. 5, pp. 4325-4335, 2021.

[8] B. O. Kurtaslan, O. Demirel, and S. S. K. Konakoglu, "Investigation of Selcuk University campus landscape design in terms of water efficient landscape arrangement," Journal of Environmental Protection and Ecology, vol. 20, no. 4, pp. 2130-2140, 2019.

[9] S. Pouya, B. Yılmaz, and O. Ateş, "Landscape design at university campus (example of Inonu University Campus Medico Square Landscape Design Project)," Akademik Ziraat Dergisi, vol. 8, no. 2, pp. 251-264, 2019.

[10] J. L. Yang, "Human landscape construction on campus: a case study of colleges and universities in northwestern Anhui," Journal of Landscape Research, vol. 9, no. 6, pp. 99-102, 2017.

[11] E. Peker and A. Ataöv, "Exploring the ways in which campus open space design influences students' learning experiences," Landscape Research, vol. 45, no. 3, pp. 310-326, 2020.

[12] D. G. Özkan, E. M. Alpak, and M. Var, "Design and construction process in campus open spaces: a case study of Karadeniz Technical University," Urban Design International, vol. 22, no. 3, pp. 236-252, 2017.

[13] L. Alwis, T. Sun, and K. T. V. Grattan, "Developments in optical fibre sensors for industrial applications," Optics \& Laser Technology, vol. 78, no. 1, pp. 62-66, 2016.

[14] K. Asibu, "Modeling of conduction mode laser weldingprocess for feedback control," Journal of Manufacturing Science and Engineering(USA), vol. 122, no. 3, pp. 420-428, 2000.

[15] W. Gui, "Application of sensor and detection technology in Mechatronics System," IOP Conference Series: Materials Science and Engineering, vol. 750, no. 1, article 012111, 2020.

[16] S. B. Yang, Y. Gao, Z. Liu, and G. Zhang, "A calibration method for binocular stereo vision sensor with short-baseline based on 3D flexible control field," Optics and Lasers in Engineering, vol. 124, no. 1, article 105817, 2021.

[17] Z. Qiao, D. Hui, and J. Huang, "Research on industrial landscape reconstruction design of Baoji Shenxin Yarn Factory," Journal of Physics: Conference Series, vol. 1549, no. 2, article 022073, 2020.

[18] L. Dong, P. Y. Hao, and X. H. Sun, "Combination and application of digital landscape technology and plant landscape design," Landscape Design, vol. 4, no. 5, pp. 102-105, 2020.

[19] C. Y. Chang, E. C. Chang, and C. W. Huang, "In situ diagnosis of industrial motors by using vision-based smart sensing technology," Sensors, vol. 19, no. 24, p. 5340, 2019.
[20] J. H. Wang and Z. H. Zhou, "The 3D reconstruction method of a line-structured light vision sensor based on composite depth images," Measurement Science and Technology, vol. 32, no. 7, pp. 1361-6501, 2021.

[21] Q. B. Wu, "Regular object volume measurement scheme based on binocular stereo vision," Modern Information Technology, vol. 4, no. 18, pp. 112-114+118, 2020.

[22] Z. Karimian, L. Samiei, and F. Kazemi, "Assessment of user preferences of campus green space at Ferdowsi University of Mashhad-Iran," Iranian Journal of Horticultural Science, vol. 48, no. Special Issue, pp. 1-11, 2017.

[23] S. Zhu, "Study on landscape visual preference of high tech Industrial park," Architecture \& Culture, vol. 1, no. 5, pp. 167-169, 2019.

[24] Y. Li, X. H. Gao, and H. P. Chen, "A survey of tourist behavior research based on visual perception," Tourism Forum, vol. 13, no. 1, pp. 82-95, 2020.

[25] Y. Zhao, J. H. Lin, and Y. Liu, "Study on visual evaluation of tourist destination landscape based on eye movement experiment-a case study of Tangjia ancient town in Zhuhai," Human Geography, vol. 35, no. 5, pp. 130-140, 2020.

[26] D. Z. Yu, "Interior landscape design and research based on virtual reality technology," Journal of Physics: Conference Series, vol. 1533, no. 3, article 032038, 2020. 